\title{
Hypo-0smolar Stress Induces p75NTR Expression by Activating Sp1-Dependent Transcription
}

\author{
Alberto Ramos, Wai Chi Ho, Stephanie Forte, Kathleen Dickson, Jacqueline Boutilier, Kristy Favell, and \\ Philip A. Barker \\ Montreal Neurological Institute, McGill University, Montreal, Quebec, Canada H3A 2B4
}

\begin{abstract}
Injury-induced expression of the p75 neurotrophin receptor (p75NTR) in the CNS facilitates neuronal apoptosis and prevents neuronal regrowth, but the mechanisms regulating p75NTR expression are poorly characterized. In this study, we showed that hypo-osmolarity induces p75NTR expression in primary neurons, and, using a comparative genomics approach, we identified conserved elements in the $25 \mathrm{~kb}$ upstream sequences of the rat, mouse, and human p75NTR genes. We found that only one of these, a proximal region rich in Sp1 sites, responds to changes in hypo-osmolarity. We then showed that Sp1 DNA binding activity is increased in cells exposed to hypoosmolarity, established that hypo-osmolarity enhanced Sp1 binding to the endogenous p75NTR promoter, and showed that Sp1 is required for $\mathrm{p} 75 \mathrm{NTR}$ expression induced by hypo-osmolarity. We examined how Sp1 is regulated to effect these changes and established that $\mathrm{Sp} 1$ turnover is strongly inhibited by hypo-osmolarity. We propose that stress-induced Sp1 accumulation that results from reductions in Sp1 turnover rate contributes to injury-induced gene expression.
\end{abstract}

Key words: neurotrophin; injury; proNGF; proteosome; promoter; osmolarity

\section{Introduction}

The p75 neurotrophin receptor (p75NTR) has emerged as a key player in the regulation of neuronal cell death and regeneration. p75NTR is highly expressed during development, but in most adult tissues, p75NTR is present at low levels (Roux and Barker, 2002). However, injury-induced p75NTR expression has been observed in many CNS pathologies, including traumatic brain injury, seizure, ischemia, and kainate-induced excitotoxity (Kokaia et al., 1998; Lopez et al., 1998; King et al., 2000; Andsberg et al., 2001; Casha et al., 2001; Copray et al., 2003). There is a tight correlation between induced p75NTR expression and subsequent apoptosis of central neurons (Roux et al., 1999), suggesting that regulation of injury-induced p75NTR expression is important for determining the degree of cell loss after injury. Consistent with this, mice rendered null for p75NTR show a sharp reduction in injury-induced apoptosis (Troy et al., 2002).

The molecular events that regulate p75NTR expression in the injured nervous system remain unknown. The structure of the p75NTR proximal promoter resembles a housekeeping gene, with no TATA or CAAT elements, a high GC content, and mul-

Received June 5, 2006; revised Dec. 21, 2006; accepted Dec. 28, 2006.

This work was supported by Canadian Institute of Health Research (CIHR) Grant MOP37850. A.R. and W.C.H. were supported by Jean Timmins Costello Foundation Fellowships, S.F. was supported by a CIHR studentship, and K.F. was supported by a Natural Sciences and Engineering Research Council Graduate Scholarship. P.A.B. is an Investigator of the CIHR. We thank Dr. Guntram Suske (Philipps University, Madeburg, Germany), Dr. Gerard Thiel (University of the Saarland Medical Center, Homburg, Germany), and Dr. Moses Chao (Cornell University, New York, NY) for providing plasmids.

Correspondence should be addressed to Philip A. Barker, Montreal Neurological Institute, McGill University, 3801 University Street, Montreal, Quebec, Canada H3A 2B4. E-mail: phil.barker@mcgill.ca.

A. Ramos's present address: Institute of Biological and Cellular Neuroscience, Faculty of Medicine, University of Buenos Aires, Calle Paraguay 21553 er piso, Buenos Aires, Argentina.

DOI:10.1523/JNEUROSCI.4806-06.2007

Copyright $\odot 2007$ Society for Neuroscience $\quad$ 0270-6474/07/271498-09\$15.00/0 tiple Sp1 binding sites (Sehgal et al., 1988; Patil et al., 1990). In transgenic mice carrying multiple copies of a $4 \mathrm{~kb}$ proximal p75NTR promoter fragment, sciatic nerve transection increased transgene expression, demonstrating that at least some of the elements controlling p75NTR expression after peripheral injury are present in the proximal $4 \mathrm{~kb}$ of the promoter (Patil et al., 1990; Huber and Chao, 1995).

Brain edema, an increase in water content of the brain parenchyma, is a common complication of traumatic brain injury, stroke, epileptic seizures, microbial infections, and brain tumors. Cytotoxic edema refers to cell swelling that results from intracellular fluid accumulation that occurs during stroke, trauma, or hypoxia (Papadopoulos et al., 2004), and this can be reproduced in vitro by exposing cells to extracellular hypo-osmolarity (Fischer et al., 1997; Caprini et al., 2003). Several genes, including p75NTR, are activated in response to this treatment (Husson et al., 1996; Meisse et al., 1998; Michalke et al., 2000; Warskulat et al., 2001; Peterson and Bogenmann, 2003), but the mechanisms that support this response and its physiological significance remain uncertain. p75NTR is almost invariably expressed after tissue injury that is accompanied by edema, and Peterson and Bogenmann (2003) have proposed that injury-induced reductions in extracellular osmolarity activate p75NTR transcription in vivo. The purpose of this study was to determine whether extracellular osmolarity regulates p75NTR expression in neurons and, if so, to identify the mechanisms that regulate this effect.

We demonstrate that hypo-osmolarity increases p75NTR mRNA and protein levels in primary mouse cortical neurons and in cell lines derived from humans, rats, and mice. To identify promoter regions that mediate this effect, we compared $25 \mathrm{~kb}$ of human, rat, and mouse promoters for conserved elements and characterized these for their response to hypo-osmolarity. The 
most proximal region, which is GC rich and contains multiple Sp1 sites, was the only element activated by hypo-osmolarity. Using electrophoretic mobility shift assays (EMSAs), we show that Sp1 DNA-binding activity is greatly enhanced by hypoosmolarity and, using chromatin immunoprecipitation (ChiP) experiments, demonstrate that hypo-osmolarity induces the association of endogenous Sp1 with the $\mathrm{p} 75 \mathrm{NTR}$ promoter. We use a variety of loss-of-function approaches to show that increased Sp1 activity is required for p75NTR expression induced by hypoosmolarity. Finally, we address the mechanism by which hypoosmolarity induces Sp1 activity and show that hypo-osmolarity increases cellular Sp1 levels by reducing its rate of degradation.

\section{Materials and Methods}

Reagents and plasmids. Antibodies directed against Sp1, Sp3, acetylated lysine, and neurotrophin receptor-interacting MAGE homolog (NRAGE) were obtained from Millipore (Bedford, MA), antibodies against actin were obtained from MP Biomedicals (Irvine, CA), and the anti-sortilin antibody was a generous gift from Dr. Andreas Nykjaer (Aarhus University, Aarhus, Denmark). The polyclonal p75NTR antibody used in these studies (termed $\alpha \mathrm{P} 1$, catalog \#07-476; Millipore) has been characterized previously using plasmid and in vivo transgenic expression studies in wild-type and p75NTR null mice and using RNA interference (RNAi) loss-of-function approaches (Majdan et al., 1997; Bhakar et al., 2003; Paul et al., 2004). Horseradish peroxidase-conjugated secondary antibodies were purchased from Jackson ImmunoResearch (West Grove, PA). All other reagents were from Sigma (St. Louis, MO), Calbiochem (La Jolla, CA), or MP Biomedicals, unless otherwise indicated. Expression plasmids for full-length $\mathrm{Sp} 1$ and $\mathrm{Sp} 3$ were obtained from Dr. Guntram Suske (Philipps University, Magdeburg, Germany), and expression plasmids pEBGN and DN-pEBGN-Sp1 were obtained from Dr. Gerard Thiel (University of the Saarland Medical Center, Homburg, Germany). Sp1 small interfering RNA was obtained from Santa Cruz Biotechnology (Santa Cruz, CA). The pGL3 basic luciferase reporter plasmid was obtained from Promega (Madison, WI).

Cell culture and transfections. HEK293T cells were purchased from American Type Culture Collection (Manassas, VA) and maintained in $5 \% \mathrm{CO}_{2}$ at $37^{\circ} \mathrm{C}$ in DMEM supplemented with $10 \%$ fetal calf serum, 2 $\mathrm{mm}$ L-glutamine, and $100 \mathrm{mg} / \mathrm{ml}$ penicillin/streptomycin. PC12 cells were maintained in $5 \% \mathrm{CO}_{2}$ at $37^{\circ} \mathrm{C}$ in DMEM supplemented with $5 \%$ bovine calf serum, $5 \%$ horse serum, $2 \mathrm{~mm}$ L-glutamine, and $100 \mathrm{mg} / \mathrm{ml}$ penicillin/streptomycin. Cortical cultures were prepared from embryonic day 15-16 mouse telencephalon; after removing meninges, neurons were dissociated in HBSS supplemented with trypsin, plated onto polyL-lysine-coated plates, and maintained in Neurobasal medium supplemented with $0.5 \times \mathrm{B} 27$ supplement and $0.5 \times \mathrm{N} 2$ supplement (both from Invitrogen, San Diego, CA), 2 mm L-glutamine, and $100 \mathrm{mg} / \mathrm{ml}$ penicillin/streptomycin for $5 \mathrm{~d}$ before use in experiments.

Immunoblotting and immunoprecipitation. For immunoblotting, cells were lysed in Nonidet P-40 (NP-40) lysis buffer (10 mm Tris, pH 8.0, 150 $\mathrm{mm} \mathrm{NaCl}, 1 \% \mathrm{NP}-40,10 \%$ glycerol) supplemented with a protease inhibitor mixture (Roche Products, Welwyn Garden City, UK), phenylmethylsulfonyl (PMSF; $1 \mathrm{~mm}$ ), and sodium vanadate (1 mM). For immunoprecipitation or nuclear protein detection, cells were lysed in Totex buffer (20 mм HEPES, pH 7.9, $350 \mathrm{~mm} \mathrm{NaCl}, 20 \%$ glycerol, $1 \% \mathrm{NP}-40$, $1 \%$ SDS, 1 mм MgCl2, 0.5 mм EDTA, 0.1 mm EGTA, $10 \mathrm{~mm} \mathrm{NaF)}$ supplemented as above. Protein concentration was determined using a BCA assay (Pierce, Rockford, IL), and samples were normalized for protein content before immunoprecipitation or immunoblotting. For immunoprecipitations, lysates were incubated with primary antibodies overnight at $4^{\circ} \mathrm{C}$ and then incubated with protein $\mathrm{A}$ agarose (for rabbit polyclonal primary antibodies) or protein $\mathrm{G}$ agarose (for mouse monoclonal primary antibodies) at $4^{\circ}$ for $1 \mathrm{~h}$. Immunocomplexes were washed extensively with NP-40 lysis buffer and eluted with sample buffer. For immunoblotting, lysate samples were separated by SDS-PAGE and transferred onto a nitrocellulose membrane. Membranes were blocked in TBST (10 mм Tris, pH 8.0, $150 \mathrm{~mm} \mathrm{NaCl,} \mathrm{2 \%} \mathrm{Tween} \mathrm{20)} \mathrm{supplemented}$ with $5 \%(w / v)$ dried skim milk powder. Primary and secondary antibody incubations were performed in blocking solution, and washes were performed using TBST. Immunoreactive bands were detected using enhanced chemiluminescence. For pulse-chase analyses, cells were preincubated in methionine-free medium for $30 \mathrm{~min}$ and maintained in medium supplemented with $50 \mu \mathrm{Ci} / \mathrm{ml}^{35} \mathrm{~S}$-methionine and $3 \mathrm{mg} / \mathrm{L}$ unlabeled methionine for $2 \mathrm{~h}$. Cells were either immediately harvested into Totex buffer for Sp1 immunoprecipitation or were incubated in nonradioactive normal or hypo-osmolar medium for $6 \mathrm{~h}$ and harvested for immunoprecipitation.

EMSA. After exposure to normal osmolarity or hypo-osmolarity, cells were washed in cold TBS, collected, and centrifuged at $1500 \mathrm{rpm}$ for 5 min. Cells were then washed in buffer A [in mM: 10 HEPES, pH 7.9, 10 $\mathrm{KCl}, 1.5 \mathrm{MgCl}_{2}, 0.5$ dithiothreitol (DTT), 0.5 PMSF], lysed in the same buffer added with $0.1 \% \mathrm{NP}-40$, and centrifuged at $13,000 \mathrm{rpm}$ for $10 \mathrm{~min}$ The pellet was resuspended in buffer B (20 mM HEPES, pH 7.9, 25\% glycerol, $420 \mathrm{~mm} \mathrm{NaCl}, 1.5 \mathrm{~mm} \mathrm{MgCl}_{2}, 0.2 \mathrm{~mm}$ EDTA, $0.5 \mathrm{~mm}$ DTT, 0.5 $\mathrm{mM}$ PMSF, $5 \mathrm{mg} / \mathrm{ml}$ aprotinin, $5 \mathrm{mg} / \mathrm{ml}$ leupeptin, $5 \mathrm{mg} / \mathrm{ml}$ pepstatin, 0.5 mu spermidine, $0.15 \mathrm{~mm}$ spermine, $100 \mathrm{~mm}$ sodium orthovanadate) and centrifuged at 13,000 rpm for $10 \mathrm{~min}$. The supernatant from this centrifugation was added to 5 vol of buffer C (20 mm HEPES, pH 7.9, 20\% glycerol, $0.2 \mathrm{~mm}$ EDTA, $50 \mathrm{~mm} \mathrm{KCl,} 0.5 \mathrm{~mm}$ DTT, $0.5 \mathrm{~mm}$ PMSF), and protein concentrations were determined. Ten milligrams of HEK293T extract or $5 \mathrm{mg}$ of cortical extract were incubated with poly dI-dC (1.5 $\mathrm{mg}$ ) for $10 \mathrm{~min}$, incubated with $0.2 \mathrm{ng}$ of DNA probe end-labeled with $\left[{ }^{32} \mathrm{P}\right]$ dNTPs, and separated on a $5 \%$ polyacrylamide gel. The gel was dried and analyzed with autoradiography. The DNA binding sequence used was ATTCGATCGGGGCGGGAG (Sp1 consensus) or ATTCGATCGGTTCGGGAG (mutated Sp1). For supershift studies, $3 \mathrm{mg}$ of the Sp1 or Sp3 antibody was incubated with samples after addition of poly dI-dC.

Reporter constructs and transcriptional assays. Distal p75NTR promoter fragments were amplified from human genomic DNA by PCR, cloned into Zero-blunt TOPO (Invitrogen), and subcloned into the pGL3 Basic Luciferase Reporter (Promega). Proximal fragments were subcloned from a plasmid containing the proximal $4 \mathrm{~kb}$ of the human p75NTR promoter (generously provided by Dr. Moses Chao, New York University, New York, NY) and subcloned into pGL3 Basic. Sequences of subcloned promoter fragments were all verified by sequencing. For transcriptional reporter assays, HEK293 cells were transfected with pGL3 constructs using Lipofectamine 2000 (Invitrogen), and $1 \mathrm{~d}$ later, cells were exposed to normal osmolarity or hypo-osmolarity for 2-24 h and lysed using buffer supplied with the Luciferase Assay System (Promega). After the protein assay, luciferase assays were performed on at least four wells per condition using the manufacturer's instructions. Samples were read by a luminometer programmed to perform a $2 \mathrm{~s}$ measurement delay and a $10 \mathrm{~s}$ measurement reading period and normalized for protein content.

Reverse transcriptase-PCR. HEK293T cells or primary cortical neurons were exposed to normal osmolarity or hypo-osmolarity for 2 or $24 \mathrm{~h}$, and mRNA was isolated using the RNAeasy Mini kit, according to the manufacturer's instructions (Qiagen, Hilden, Germany). cDNA was generated using the Omniscript RT kit (Qiagen) with random hexamers (Roche Products), and PCR was performed using primers specific for p75NTR (human, forward 5' -TACGGCTACTACCAGGATGAGACG3', reverse 5'-CCAGGATGGAGCAATAGACAGG-3', 448 bp product; mouse, forward $5^{\prime}$-TATGGCTACTACCAGGACGAGG, reverse $5^{\prime}$ CCAAGATGGAGCAATAGACAGG, 448 bp product) or actin (forward 5'-CACCACTTTCTACAATGAGC, reverse 5'-CGGTCAGGATCTTCATGAGG, $322 \mathrm{bp}$ product). PCR products were run in a $1.5 \%$ agarose gel and photographed in a Bio-Rad (Hercules, CA) VersaDoc 4000 imaging system. Each reverse transcriptase-PCR (RT-PCR) experiment was run with negative control reactions in which Omniscript reactions were performed in the absence of reverse transcriptase; the negative controls consistently failed to generate a PCR product (data not shown).

ChIP assay. After exposing cells to normal osmolarity or hypoosmolarity for $4 \mathrm{~h}$, formaldehyde was added to the medium ( $1 \%$ final concentration) for $10 \mathrm{~min}$ to cross-link cellular proteins and DNA. Cells were washed with cold PBS, collected in PBS, and centrifuged at 1500 $\mathrm{rpm}$ for $5 \mathrm{~min}$. Cells were lysed with buffer B1 (50 mM Tris, $1 \mathrm{~mm}$ EDTA, 
$120 \mathrm{~mm} \mathrm{NaCl}, 0.1 \% \mathrm{NP}-40,10 \%$ glycerol) and centrifuged at $3000 \mathrm{rpm}$ for $5 \mathrm{~min}$. The nuclei were resuspended in buffer B2 (50 mM Tris, $1 \%$ SDS, 1 mm EDTA) and sonicated using a Branson Sonifier 450 (three pulses with $12 \mathrm{~s}$ each). Lysate was centrifuged at $13,000 \mathrm{rpm}$ for 10 min, diluted 10 times with buffer B3 (16.7 mM Tris, $167 \mathrm{~mm} \mathrm{NaCl}, 1.2 \mathrm{~mm}$ EDTA, 1.1\% Triton $\mathrm{X}-100,0.01 \%$ SDS), and precleared with salmon sperm DNA/protein A agarose (Upstate Biotechnology, Lake Placid, NY) at $4^{\circ} \mathrm{C}$ for $1 \mathrm{~h}$. One hundred microliters of each cleared sample were retained as total input controls. Immunoprecipitation was performed using $5 \mu \mathrm{g}$ of Sp1 antibodies (Upstate Biotechnology) at $4^{\circ} \mathrm{C}$. The complexes were then washed once with low-salt washing buffer $(20 \mathrm{~mm}$ Tris, 2 mм EDTA, 0.1\% SDS, $1 \%$ Triton X-100, 150 $\mathrm{mm} \mathrm{NaCl})$, once with high-salt buffer $(20 \mathrm{~mm}$ Tris, 2 mм EDTA, $0.1 \%$ SDS, $1 \%$ Triton X-100, $500 \mathrm{~mm} \mathrm{NaCl})$, once with $\mathrm{LiCl}$ buffer $(0.25 \mathrm{M}$ LiCl, 1\% NP-40, 1\% deoxycholic acid, $1 \mathrm{~mm}$ EDTA, $10 \mathrm{~mm}$ Tris), and three times with TE buffer (10 mm Tris and $1 \mathrm{~mm}$ EDTA). The complexes were eluted with freshly prepared extraction buffer ( $1 \%$ SDS, $\left.0.1 \mathrm{M} \mathrm{NaHCO}_{3}\right)$. Protein/ DNA cross-linking was reversed by incubation at $65^{\circ} \mathrm{C}$ overnight, and the DNA was purified using a PCR purification kit (Qiagen). The purified DNA and input genomic DNA were then subjected to PCR using primers specific for the proximal Sp1-rich region of the p75NTR promoter.

Hypo-osmolar exposure. Hypo-osmolar medium was prepared by diluting medium with sterile water to obtain the desired osmolarity and was confirmed with an osmometer (Advanced Instruments, Needham Heights, MA) (Peterson and Bogenmann, 2003). For normal controls, water used for medium dilution was replaced by a sterile isotonic $\mathrm{NaCl}$ solution $(0.9 \% \mathrm{w} / \mathrm{v})$. For neuronal cultures, hypoosmolar medium was prepared by dilution of Neurobasal medium while maintaining $1 \times$ concentration of B27, glutamine, and penicillin/streptomycin concentration constant.

Sequence analysis. Sequences of the human, rat, and mouse p75NTR promoters were obtained from the University of California Santa Cruz (Santa Cruz, CA) genome browser (http:// genome.ucsc.edu) using genome assemblies from February 2003 (mouse), June 2003 (rat), and July 2003 (human). Twenty-five kilobases upstream from the initial point of transcription were analyzed simultaneously using the MultiPip Marker program (http://pipmaker.bx.psu.edu), setting the single coverage option to exclude interspersed repeats.

Statistical analysis. Quantitative data were analyzed by one-way ANOVA, with probabilities assessed using the Tukey-Kramer multiple comparisons test.

\section{Results}

\section{p75NTR mRNA and protein levels} are increased by exposure to hypo-osmolarity

To confirm that reducing extracellular solute concentration can induce the expression of p75NTR, we tested the effect of hypoosmolarity on p75NTR expression on a variety of primary and cultured cells. Both HEK293T cells (Fig. 1A) and primary mouse cortical neurons (Fig. $1 B$ ) express little p75NTR under normal growth conditions but exhibit robust p75NTR expression when extracellular osmolarity is reduced. In HEK293 cells, increased
B

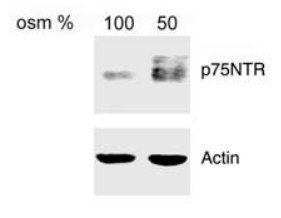

C

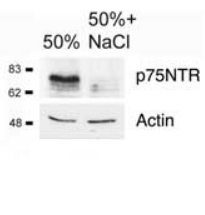

$E$
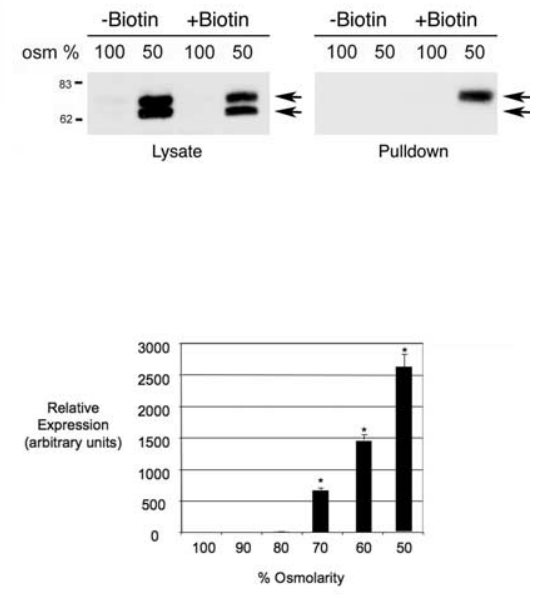

Figure 1. Exposure to hypo-0smolarity increases p75NTR expression in cell lines and in primary mouse cortical neurons. $\boldsymbol{A}$, HEK293 cells were exposed to increasing hypo-osmolarity for $24 \mathrm{~h}$, lysed, and analyzed for p75NTR and actin expression by Recovery) for the times indicated. After lysis, cells were analyzed for p75NTR and actin expression by immunoblot. Ctl, Control. $\boldsymbol{E}$ noblots of lysates (left) and streptavidin pulldowns (right) are shown. Arrows indicate the isoforms of p75NTR induced by hypo.F, Left, HEK293 cells were exposed to decreasing osmolarity for $24 \mathrm{~h}$, lysed, and analyzed by immunoblot for levels o f the experiment $\left({ }^{*} p<0.05\right.$, significant difference in $p 75$ NTR levels compared with $24 \mathrm{~h}$ exposure to normal medium). Experiments shown in $\boldsymbol{A}-\boldsymbol{E}$ were repeated three times with identical results. osm, 0smolarity.

p75NTR protein levels were induced by $65 \%$ osmolarity and were maximal at 50\%, whereas actin levels remained unchanged under these conditions. Although HEK293 cells exposed to hypotonic medium showed robust p75NTR induction, when supplemented to isotonicity with $\mathrm{NaCl}$, hypotonic medium had no effect on p75NTR expression (Fig. 1C), demonstrating that changes in p75NTR expression are induced by reduced extracellular osmolarity and not by dilution of a specific medium component. Other cell lines (A875 melanoma, U373 glioma, MDA231 breast cancer, PC12 pheochromacytoma) exhibited similar, although less robust, responses (data not shown). We therefore focused our remaining studies on primary mouse cortical neurons and HEK293T cells.

Hypo-osmolarity induced p75NTR protein expression in HEK293 cells within $12 \mathrm{~h}$ (Fig. 1D). p75NTR initially produced $\sim 55-70 \mathrm{kDa}$, and the $75 \mathrm{kDa}$ isoform of p75NTR began to accumulate by $18 \mathrm{~h}$. This process was reversible; switching cells back to normal medium led to a decline in p75NTR levels that left only baseline p75NTR levels $48 \mathrm{~h}$ after the switch. The time course of induction of the lower and higher molecular weight isoforms of 


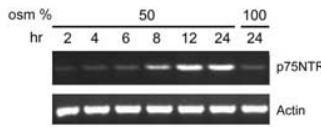
Relative
Expression
(arbitrary units)
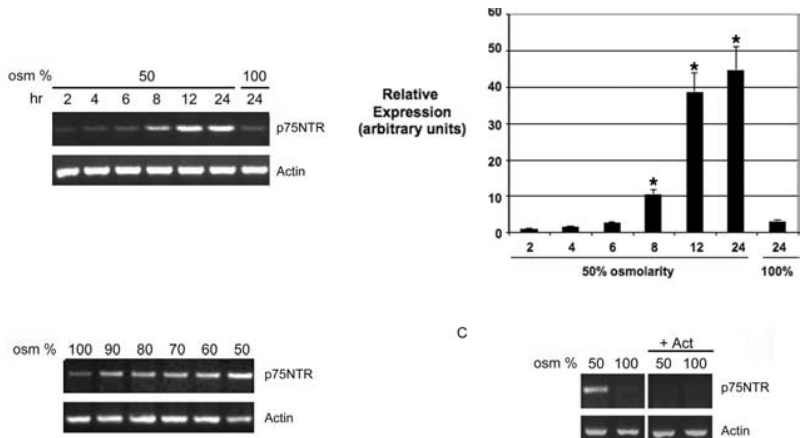

c
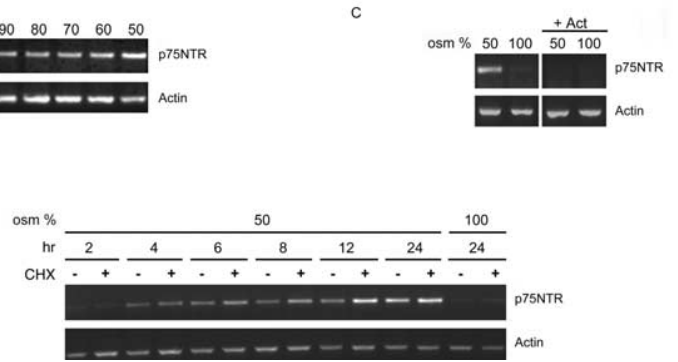

Figure 2. Hypo-osmolarity increases p75NTR mRNA levels. $\boldsymbol{A}, \boldsymbol{B}$, Primary mouse cortical neurons were exposed to $50 \%$ hypo-0smolar medium for different periods of time $(\boldsymbol{A}$, left) or to different levels of hypo-osmolarity $(\boldsymbol{B})$. RNA was extracted, and p75NTR and actin mRNAs were evaluated by RT-PCR, as described in Materials and Methods. The increase in p75NTR mRNA levels shown in $\boldsymbol{A}$ was quantified by densitometry of three repeats of the experiment (right) $\left({ }^{*} p<0.05\right.$, significant difference in p75NTR levels compared with $24 \mathrm{~h}$ exposure to normal medium). C, Primary mouse cortical neurons were exposed to hypo-osmolarity ( $50 \%$ osm) or normal medium ( $100 \%$ osm) for $24 \mathrm{~h}$ in the presence or absence of actinomycin (Act; $1 \mu \mathrm{g} / \mathrm{ml}$ ), and P75NTR and actin mRNAs were evaluated by RT-PCR. D, Primary mouse cortical neurons were exposed to hypo-osmolarity ( $50 \%$ osm) or normal medium (100\% osm) for $24 \mathrm{~h}$ in the presence or absence of cycloheximide (CHX; $10 \mu \mathrm{g} / \mathrm{ml})$, and p75NTR and actin mRNAs were evaluated by RT-PCR. All experiments shown were repeated three times. osm, 0smolarity.

p75NTR suggested that the lower band represents immature p75NTR present in intracellular compartments and that the higher molecular form represents p75NTR at the cell surface. To address this, cell-surface biotinylation assays were performed on untreated cells and on cells exposed to hypo-osmolarity; Figure $1 E$ shows that only the $75 \mathrm{kDa}$ isoform of p75NTR is present at the cell surface, indicating that the lower molecular weight forms likely represent immature p75NTR in the synthetic pathway.

To examine the specificity of this response, we examined whether expression of the p75NTR coreceptor Sortilin, the p75NTR adaptor protein NRAGE, or the p75NTR homolog $\mathrm{NRH} 2$ were altered by hypo-osmolarity and found that none of these were induced by this treatment (Fig. $1 F$ ).

Semiquantitative RT-PCR was then used to determine whether changes in p75NTR protein levels were reflected in alterations in p75NTR mRNA. In primary cortical neurons, hypoosmolarity increased p75NTR mRNA levels after $6 \mathrm{~h}$ of exposure, and this peaked within $12 \mathrm{~h}$ (Fig. $2 \mathrm{~A}$ ). The induction of p75NTR was observed with only a $10 \%$ reduction in osmolarity, indicating that p75NTR expression in these neurons is altered by modest changes in extracellular solute concentration (Fig. $2 \mathrm{~B}$ ). Figure $2 \mathrm{C}$ shows that actinomycin blocked the increase in p75NTR mRNA, indicating that its accumulation required transcription. Cycloheximide did not block p75NTR mRNA induction, demonstrating that new protein synthesis is not required for this effect (Fig. 2D). We examined the same parameters in HEK293 cells and found that time course, dose responsiveness, and the effect of transcription and translation were essentially identical to those observed in cortical neurons (data not shown).
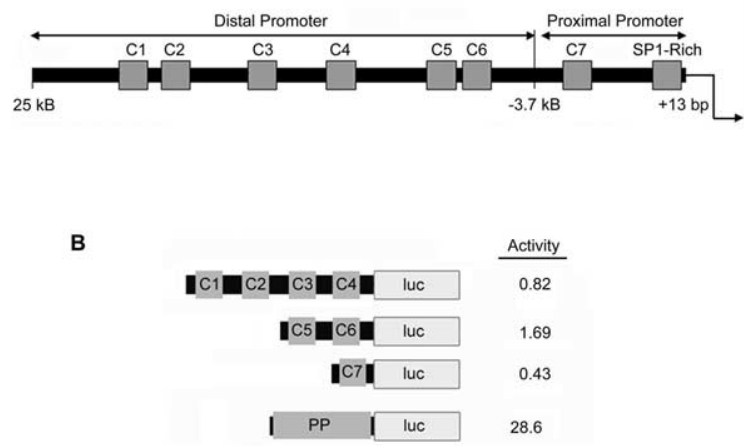

C

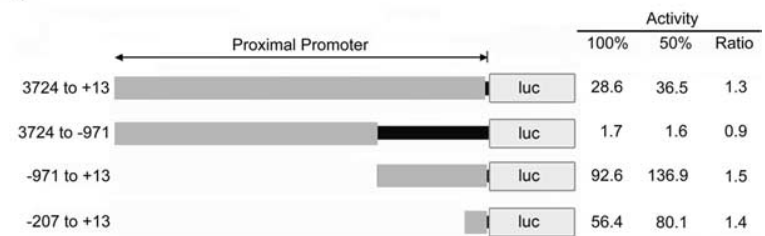

Figure 3. The p75NTR promoter contains cis-elements that respond to hypo-osmolarity. $A, A$ schematic representation of the relative positions of conserved regions in the p75NTR promoter. Boxes $\mathrm{C} 1$ to $\mathrm{C} 7$ and the proximal Sp1-rich region represent regions of $>100$ bp that contain at least $65 \%$ homology between mouse, rat, and human. $\boldsymbol{B}$, The conserved fragments were cloned into a PGL3-Basic reporter and used to transfect HEK293 cells. Twenty-four hours after transfection, cells were exposed to either hypo-osmolar or normal medium for $18 \mathrm{~h}$, lysed, and assayed for luciferase activity. The ratio of activity in hypo-osmolarity versus normal medium is presented. PP, Proximal promoter. $\boldsymbol{C}$, The $3.7 \mathrm{~kb}$ proximal promoter and the indicated subdomains were assayed for luciferase activity as in $\boldsymbol{B}$. The ratio of activity in hypo-osmolarity versus normal medium is presented. Experiments in $\mathbf{C}$ were performed four times with identical results, using triplicates or quadruplicates for each sample in each experiment. luc, Luciferase.

\section{The p75NTR promoter contains cis-elements that respond} to hypo-osmolarity

Regulatory elements in eukaryotic promoters are highly conserved and can be identified by comparative genomics (Dickmeis and Muller, 2005). The hypo-osmolarity-induced p75NTR expression is observed in cell types derived from mice, rats, and humans, and it is reasonable to assume that cis-acting regulatory sequences responsible for this effect are conserved between these species. Therefore, we compared the $25 \mathrm{~kb}$ upstream regions of the $p 75 N T R$ gene for putative conserved regulatory sequences $>100$ bp and at least $65 \%$ homologous between these three species (Nobrega and Pennacchio, 2004). Eight conserved fragments that met these criteria were identified. One of these was the Sp1rich region of the p75NTR that lies directly upstream of the p75NTR transcriptional start site (Patil et al., 1990), and the others [designated C1 (most distal) to C7 (most proximal)] were located in a region encompassing -2800 to $-16,900 \mathrm{bp}$ from the transcriptional start site (Fig. $3 A$ and supplemental Fig. 1, available at www.jneurosci.org as supplemental material).

To determine whether these conserved cassettes respond to changes in hypo-osmolarity, they were subcloned, individually and in tandem, into a plasmid containing a minimal SV40 promoter driving luciferase expression and analyzed for their ability to respond to changes in hypo-osmolarity. The conserved regions $\mathrm{C} 1$ to $\mathrm{C} 7$ conferred low rates of basal transcriptional activity in the reporter constructs but showed no response to hypoosmolarity, even when the treatment was applied for extended periods (Fig. 3B) (data not shown). In contrast, the Sp1-rich 
A

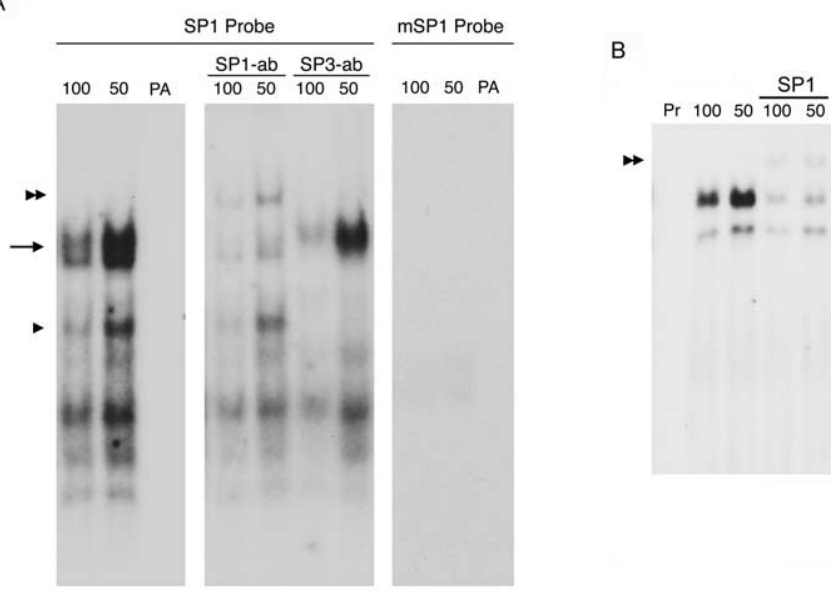

C

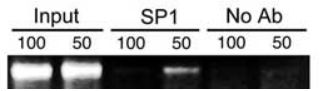

Figure 4. Sp1 DNA binding activity is increased in hypo-osmolar conditions. $\boldsymbol{A}, \boldsymbol{B}, \mathrm{HEK} 293$ cells $(\boldsymbol{A})$ or primary cortical neurons $(\boldsymbol{B})$ were exposed to hypo-osmolarity ( $50 \%$ osm) or normal osmolarity (100\% osm) for $4 \mathrm{~h}$, and the Sp1 binding activity was evaluated by EMSA. Antibodies directed against Sp1 (Sp1-ab) or Sp3 (SP3-ab) were used to supershift complexes bound to the Sp1 element. Major and minor complexes referred to in the text are indicated by the arrow and single arrowhead, respectively. Note that only small amounts of the supershifted complexes are present (double arrowheads), likely because these protein-DNA complexes are too large to enter the gel. The rightmost panel in $\boldsymbol{A}$ shows EMSAs performed using a labeled mutant Sp1 probe (mSP1 probe). C, HEK293 cells were exposed to hypo-0smolarity (50\% osm) or normal osmolarity ( $100 \%$ osm) for $4 \mathrm{~h}$, and the level of Sp 1 bound to the endogenous p75NTR promoter was evaluated by CHIP assay. Experiments shown in $\boldsymbol{A}$ and $\boldsymbol{B}$ were repeated four times, and the experiment shown in $C$ was repeated three times with identical results. $\mathrm{PA}$, Probe alone; $\mathrm{Pr}$, probe; Ab, antibody.

proximal region had higher levels of basal activity, and luciferase production was significantly increased by reducing extracellular osmolarity. We therefore focused our efforts on this region. Deletion analysis of this proximal promoter fragment allowed us to identify a 200 bp subdomain, designated $\mathrm{PP}^{200}$, that accounted for essentially all of the responsive hypo-osmolarity contained within the proximal promoter (Fig. $3 C$ ). $\mathrm{PP}^{200}$ is located at positions -207 to +13 (relative to the p75NTR transcriptional start site), is CG rich, and contains seven putative Sp1 binding sites.

\section{Sp1 is required for p75NTR expression induced}

\section{by hypo-osmolarity}

Sp1 is typically considered an unregulated transcription factor important for constitutive expression of a variety of housekeeping genes. However, the presence of numerous Sp1 binding sites in the $\mathrm{PP}^{200}$ fragment that is responsive to hypo-osmolarity prompted us to test the possibility that hypo-osmolarity increases Sp1 activity at the p75NTR promoter and thereby enhances p75NTR transcription rates. To test this, we performed EMSAs on nuclear extracts derived from HEK293T cells or primary cortical neurons that had been exposed to hypo-osmolarity for 2 or 4 h. Sp1 binding activity was substantially increased in HEK293 cells (Fig. 4A) or in cortical neurons (Fig. 4B) exposed to hypoosmolarity. In contrast, a mutated Sp1 binding element incapable of binding $\mathrm{Sp} 1$ did not form shifted complexes in the absence or presence of hypo-osmolarity (Fig. $4 A$, right).

Sp1 and the related factor Sp3 bind the same consensus sequence, and we therefore performed supershift analyses to iden- tify the specific Sp1 family members that were regulated by hypoosmolarity. In HEK293 cells, antibodies directed against Sp1 supershifted the major complex induced by hypo-osmolarity but had no effect on two minor slower migrating complexes, whereas SP3 antibodies supershifted the minor complexes but not the major complex (Fig. 4A). In neurons, Spl antibodies supershifted both of the complexes induced by hypo-osmolarity (Fig. $4 B$ ). Together, these data suggest that transcription factor binding to $\mathrm{Sp} 1$ sites increases with hypo-osmolarity and that the Sp1 protein is the predominant component of this binding activity.

To determine whether the increase in Sp1 binding activity observed in EMSA translates into enhanced Sp1 binding at the p75NTR promoter in intact cells, ChIP assays were performed (Fig. 4C). HEK293 cells were left untreated or exposed to hypoosmolarity for $4 \mathrm{~h}$, and antibodies directed against Sp1 were used to immunoprecipitate protein-DNA complexes. PCR analysis of the isolated DNA fragments indicates that hypo-osmolarity causes a significant increase in the amount of $S p 1$ associated with the p75NTR promoter. We also used ChIP analyses to examine Sp3 binding to the $\mathrm{p} 75 \mathrm{NTR}$ promoter, but these experiments did not detect a significant association (data not shown).

To establish whether Sp1 plays a role in the induction of p75NTR that occurs in response to hypo-osmolarity, we used three loss-of-function approaches. First, we asked whether a dominant-negative form of Sp1 that has an intact DNA binding domain but lacks the transactivation domain (DN-Sp1) can interfere with induced p75NTR expression. Overexpression of DNSp1 strongly reduced the increase in p75NTR protein and p75NTR mRNA that normally occur in response to hypoosmolarity (Fig. 5A,B). Next, we used RNAi directed against Sp1 to reduce endogenous Spl levels and assessed its effect on p75NTR expression. Figure 5C shows that RNAi directed against Sp1 significantly reduced Sp1 expression, whereas a corresponding scrambled oligonucleotide did not. The reduction in Sp1 levels attenuated the induction of p75NTR induced by hypoosmolarity, indicating that Sp1 is required for this response. Finally, we treated HEK293 cells and primary cortical neurons with mithramycin A, an aureolic antibiotic that selectively inhibits Sp1-mediated transcriptional activation, and found that this compound strongly reduced p75NTR expression induced by hypo-osmolarity in these cell types (Fig. $5 D, E$ ). These results indicate that the increase in $\mathrm{p} 75 \mathrm{NTR} \mathrm{mRNA}$ and protein induced by hypo-osmolarity requires $\mathrm{Sp} 1$ activity.

\section{Hypo-osmolarity increases $\mathrm{Sp} 1$ levels by reducing its turnover rate}

The increase in p75NTR mRNA induced by hypo-osmolarity occurs in the presence of cycloheximide (Fig. 2D) and therefore likely reflects post-translational changes in Sp1 or in cofactors that regulate $\mathrm{Sp} 1$ activity. We considered the possibility that reducing extracellular osmolarity alters the subcellular location of Sp1, but fractionation studies showed that hypo-osmolarity had no effect on the cytosolic-to-nuclear ratio of Sp1 (data not shown). However, immunoblotting revealed that exposure to hypo-osmolarity caused a significant increase of cellular Sp1 protein levels (Fig. 6A). To determine whether this increase reflects hypo-osmolarity-induced Sp1 mRNA accumulation, we examined Sp1 mRNA levels by RT-PCR and found that they were not changed by this treatment (Fig. 6B).

To test the possibility that the accumulation of $\mathrm{Sp} 1$ protein reflects a reduction in its degradation rate, we first asked whether the increase in Sp1 levels occurred in cells exposed to cycloheximide. Figure $7 A$ shows that cycloheximide does not block the 

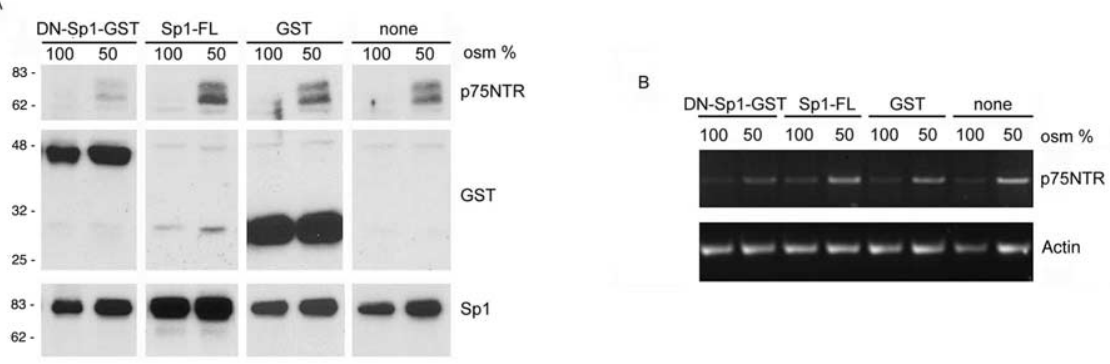

C

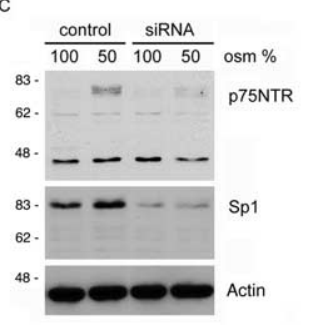

D

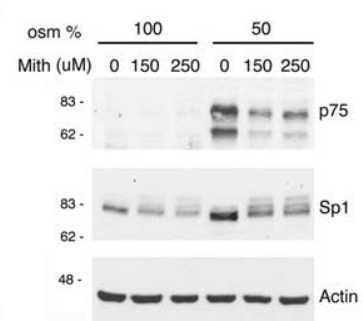

$E$

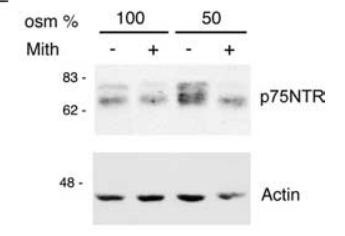

Figure 5. Sp1 is required for p75NTR expression induced by hypo-osmolarity. $\boldsymbol{A}, \boldsymbol{B}$, HEK293 cells were left untransfected (none) or were transfected with plasmids expressing dominant-negative Sp1 glutathione S-transferase (DN-Sp1-GST), full-length Sp1 (Sp1-FL), or GST alone (GST). Twenty-four hours after transfection, cells were exposed to hypo-0smolarity (50\% osm) or normal osmolarity (100\% 0sm) for $24 \mathrm{~h}$, lysed, and analyzed for levels of p75NTR, GST, and Sp1 protein by immunoblot (A) or for p75NTR and actin mRNA by RT-PCR (B). C, HEK293 cells were transfected with small interfering RNA (siRNA) directed against Sp1 or were transfected with a scrambled control siRNA. The next day, the cells were exposed to hypo-0smolarity (50\% osm) or normal osmolarity (100\% 0sm) for $24 \mathrm{~h}$, lysed, and analyzed for p75NTR, Sp1, and actin expression by immunoblot. D, HEK293 cells were exposed to hypo-osmolarity or to normal medium for $24 \mathrm{~h}$ in the presence of increasing doses of mithramycin $A$ and analyzed for p75NTR, Sp1, and actin expression by immunoblot. $\boldsymbol{E}$, Primary cortical neurons were exposed to hypo-osmolarity or to normal medium for $24 \mathrm{~h}$ in the presence of $250 \mu \mathrm{m}$ mithramycin $\mathrm{A}$ for $24 \mathrm{~h}$ and analyzed for $\mathrm{p} 75 \mathrm{NTR}$ and actin expression by immunoblot. All experiments shown were repeated three times with identical results. osm, 0smolarity; Mith, mithramycin A.

\section{A}

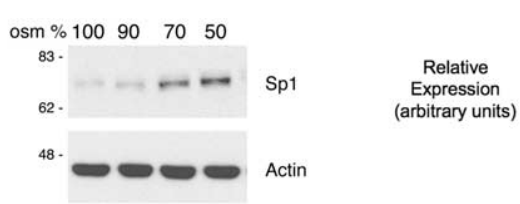

B

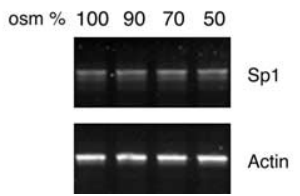

Figure 6. Sp1 levels are increased in response to hypo-0smolarity. HEK293 cells were exposed to increasing hypo-0smolarity, as indicated, for $24 \mathrm{~h}$, lysed, and analyzed for Sp1 protein expression by immunoblot $(\boldsymbol{A})$ or for Sp1 mRNA expression by RT-PCR $(\boldsymbol{B})$. The increase in Sp1 protein was quantified by densitometry of three repeats of the experiment $\left(\boldsymbol{A}\right.$, right) $\left({ }^{*} p<\right.$ 0.05 , significant difference in $\mathrm{Sp} 1$ levels compared with $24 \mathrm{~h}$ exposure to normal medium). All experiments shown were repeated three times with identical results. osm, 0smolarity.

increase of Sp1 in cells exposed to hypo-osmolarity, suggesting that hypo-osmolarity may block Spl degradation. Interestingly, cycloheximide on its own caused an increase in Spl protein, even in cells maintained in normal medium. A paradoxical increase in protein level induced by cycloheximide has been reported for several genes and is termed super-induction. (Hershko et al., 2004); mRNAs that produce this response typically contain 3-10 copies of the consensus sequence AUUUA in their 3' uncoding regions, and consistent with this, examination of this region of the human $\mathrm{Sp} 1$ mRNA revealed 7 AUUUA sequences.

To directly determine whether $\mathrm{Sp} 1$ turnover kinetics change within cells exposed to hypo-osmolarity, we used pulse-chase analyses to assess the fate of $\mathrm{Sp} 1$. For this, cells were pulsed with ${ }^{35} \mathrm{~S}$ methionine for $1 \mathrm{~h}$, chased with normal medium for 1 additional hour, and harvested or maintained for $6 \mathrm{~h}$ in normal or hypo-osmolar medium. Figure $7 B$ shows that Sp1 that was immunoprecipitated directly after exposure to ${ }^{35} \mathrm{~S}$-methionine was readily detected and that levels of labeled Sp1 were greatly reduced after a $6 \mathrm{~h}$ exposure to normal medium. Quantification by densitometry revealed that the labeled pool of Sp1 was decreased by $70 \%$ at the end of this period, demonstrating that $\mathrm{Sp} 1$ is turned over rapidly under normal conditions. However, the pool of labeled Sp1 pool levels remained stable in cells exposed to hypo-osmolarity for $6 \mathrm{~h}$, indicating that the Sp1 degradation rate is strongly attenuated in cells exposed to hypo-osmolarity.

To begin to address the signaling pathways that regulate p75NTR gene expression in response to hypo-osmolarity, we asked whether selective inhibitors of phosphoinositol-3-kinase (PI3K) [LY294002 (2-(4-morpholinyl)-8-phenyl-4H-1-benzopyran-4-one hydrochloride)], protein kinase C (PKC) [Go6976 (2-[1-(3-dimethylaminopropyl)-5-methoxyindol-3-yl]-3-(1 Hindol-3-yl)maleimide)], phospholipase C (PLC) [U73122 (1-(6[17 $\beta$-3-methoxyestra-1,3,5-(10) triene-17-yl] amino/hexyl) $1 H$ pyrroledione)], or nitric oxide synthase (NOS) [L-NAME ( $\mathrm{N} \omega$ nitro-L-arginine methyl ester)] alter this response. Figure $7 C$ shows inhibition of PI3K and PKC strongly attenuated the p75NTR induction, whereas inhibition of phospholipase enhanced the expression of p75NTR. Importantly, the effect of these inhibitors on p75NTR expression correlated with changes in Sp1 protein levels, consistent with the hypothesis that changes in Sp1 levels mediate the induction of p75NTR expression in cells exposed to hypo-osmolarity.

\section{Discussion}

Induction of p75NTR expression is a prominent feature of many types of CNS injury. The induced expression of p75NTR can result in neuronal apoptosis, or when sublethal, facilitates production of the amyloid $\beta$-peptide and increases responses of neurons to neuronal growth inhibitors that include Nogo-A and MAG (Ceni and Barker, 2005; Costantini et al., 2006). Identifying the mechanisms that facilitate the acute changes in p75NTR expression that accompany injury may provide insights with clinical impact. Edema is a rapid and almost universal response to brain injury that often has a profound pathogenic effect in the clinic. Consequences and treatment of edema have typically focused on the mechanical effects of CNS swelling, and the genetic effects of this common and often devastating condition have not been studied extensively. 
A

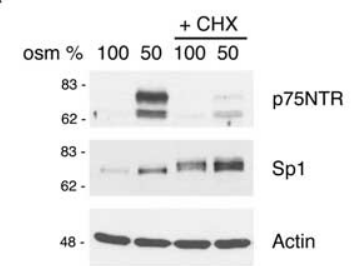

B
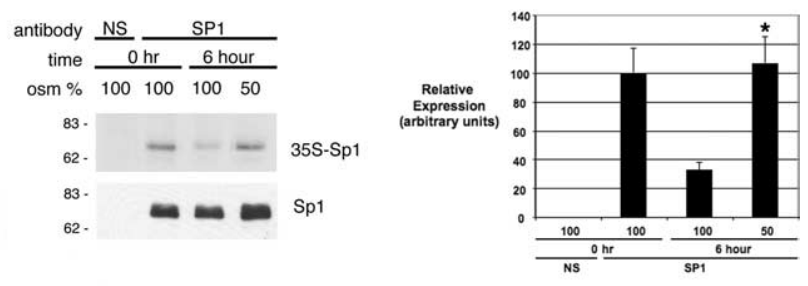

C

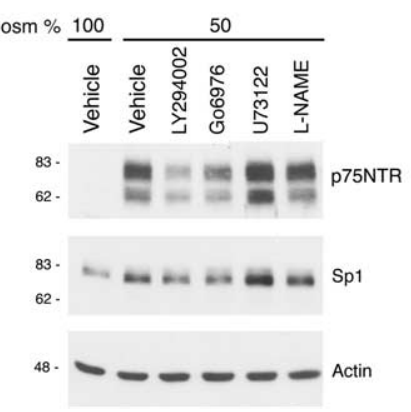

Figure 7. Exposure to hypo-osmolarity reduces Sp1 turnover. A, HEK293 cells were exposed to hypo-osmolarity ( $50 \%$ osm) or normal osmolarity ( $100 \%$ osm) in the presence or absence of cyclohexymide (CHX; $10 \mu \mathrm{g} / \mathrm{ml}$ ) for $24 \mathrm{~h}$, lysed in Totex buffer, and analyzed for p75NTR, Sp1, and actin expression by immunoblot. $\boldsymbol{B}$, Left, Sp1 turnover was assessed by pulse-chase metabolic labeling, immunoprecipitation, SDS-PAGE, and flourography, as described in Materials and Methods. Right, Changes in ${ }^{35}$ S-labeled Sp1 levels were quantified by densitometry of three repeats of the experiment ${ }^{*} p<0.05$, significant difference in $S p 1$ level in cells exposed to hypo-osmolarity vs normal osmolarity for 6 h). NS, Nonspecific antibody; Sp1, Sp1 antibody. C, HEK293 cells were exposed to hypo-osmolarity (50\%) or maintained in normal medium with normal osmolarity (100\%) for $24 \mathrm{~h}$ in the presence or absence of inhibitors directed against PI3K (LY294002), PKC (G06976), PLC (U73122), or NOS (L-NAME).

From a cellular perspective, the main change elicited by edema is a reduction in extracellular osmolarity. We have used cells maintained in vitro and exposed to hypo-osmolarity as a model system to determine whether extracellular osmolarity alters p75NTR expression and to dissect the mechanisms responsible for the changes observed. In this study, we confirm previous findings that showed that $\mathrm{p} 75 \mathrm{NTR}$ levels are increased in cell lines exposed to hypo-osmolarity (Peterson and Bogenmann, 2003) and show that this induction also occurs in primary cortical neurons. We demonstrate that the accumulation of p75NTR mRNA requires transcription but is independent of translation, occurs rapidly, and is reversible. Primary cortical neurons are particularly sensitive to the effects of hypo-osmolarity, with increased expression of p75NTR mRNA observed with only a 10\% drop in osmolarity, well within the osmolarity shift that occurs in vivo during injury-induced edema. Higher osmolarity shifts (e.g., $35 \%$ decrease) are required to detect increased $\mathrm{p} 75 \mathrm{NTR}$ protein, but this likely reflects the relative insensitivity of the immunoblotting technique used to detect the receptor. Of the cell lines examined, HEK293 cells show a particularly robust increase in
p75NTR levels in response to hypo-osmolarity; the reasons for the cell-type specificity of this response are unknown, but it is noteworthy that the originators of this line have recently concluded that HEK293 cells have a neuronal origin (Shaw et al., 2002).

The induction of p75NTR occurs in mouse, rat, and human cell lines, suggesting that conserved elements in the p75NTR promoter may subserve this effect. Cross-species comparisons of promoters have emerged as a powerful technique to identify important cis-regulatory sequences (Farhadi et al., 2003; Nobrega and Pennacchio, 2004), and we therefore compared promoters across these species to identify conserved regions in the p75NTR promoter. Seven conserved regions were identified within the 25 $\mathrm{kb}$ of sequence lying $5^{\prime}$ to the p75NTR transcriptional start site, but only the most proximal of these was responsive to hypoosmolarity. The selective pressure that forced conservation among the other promoter regions suggests that they may play some role in developmental, tissue-specific, or injury-induced p75NTR gene regulation, but the regulatory events that impinge on them remain unknown.

The proximal conserved region of the p75NTR promoter that is activated by hypo-osmolarity is GC rich and contains multiple $\mathrm{Sp} 1$ binding sites. $\mathrm{Sp} 1$ is a ubiquitous transcription factor implicated in the expression of numerous genes and is typically regarded as a constitutive transcription factor that performs housekeeping functions. When we performed experiments to rule out its involvement in the effect of hypo-osmolarity, we were surprised to learn that Spl binding to its cognate recognition sequence was strongly induced by this treatment. The related factor Sp3, which binds the same cis-element, also showed enhanced DNA binding in response to hypo-osmolarity, albeit to a lesser degree than for Sp1. To learn whether this change in the in vitro binding capacity of Sp1 corresponded to increased occupancy of the endogenous $\mathrm{p} 75 \mathrm{NTR}$ promoter, ChIP assays were performed, and these showed that Sp1 binding to the p75NTR promoter was increased substantially by treatment with hypo-osmolarity.

We showed that p75NTR expression induced by hypoosmolarity required Sp1 using several loss-of-function strategies. Overexpression of an Spl dominant-negative construct that binds GC boxes but does not mediate transactivation blocked the induction of p75NTR. Mithramycin, which blocks Sp1 binding to DNA, similarly blocked the effects of hypo-osmolarity on p75NTR expression. Both of these approaches show that ciselements that bind Sp1 play a crucial role in the induction of p75NTR, but neither approach directly implicates Sp1 in this effect. To address this, we used RNAi to reduce cellular Sp1 levels and showed that this strongly attenuated the induction of p75NTR. We therefore conclude that Sp1, functioning through GC boxes in the p75NTR promoter, plays a crucial role in the induction of p75NTR induced by hypo-osmolarity.

A prevalent view regarding $\mathrm{Sp} 1$ and $\mathrm{Sp} 3$ is that they are constituents of a core transcriptional complex that is required for basal transcription and that it functions as a "housekeeping" gene. However, over the last several years, several studies have shown that Sp1 and Sp3 transcription factors are, in fact, highly regulated by extracellular cues and that their levels and posttranslational modifications have dramatic effects on the expression of several specific genes (Bouwman and Philipsen, 2002; Li et al., 2004). Although our findings are the first to show that hypoosmolarity increases cellular levels of Sp1, these results expand on previous studies that have shown that levels of Sp1 can be induced by a variety of stimuli (Li et al., 2003; Wong et al., 2003; Pang et al., 2004). An emerging consensus is that the relative ratio of Sp1 
and Sp3 helps determine transcriptional output, and there are several instances in which a high $\mathrm{Sp} 1 / \mathrm{Sp} 3$ ratio favors increased transcription, whereas the predominance of Sp3 favors transcriptional repression (Birnbaum et al., 1995; Majello et al., 1997). Our ChIP assays established that Sp1 binding to the p75NTR promoter increases with exposure to hypo-osmolarity, but we could not detect binding of SP3 to the endogenous p75NTR promoter, either because this factor does not bind this promoter element in vivo or because the ChIP assays were insufficiently sensitive to reveal this interaction.

Sp1 levels are substantially increased in cells exposed to hypoosmolarity. We found that inhibitors of PI3K, PKC, and PLC that alter the induction of p75NTR show a corresponding effect on Sp1 levels; PI3K and PKC inhibitors block the accumulation of Sp1 and block the induction of p75NTR, whereas a PLC inhibitor enhances accumulation of Sp 1 and boosts p75NTR induction. An inhibitor of NOS activity had no effect on p75NTR accumulation or Sp1 levels. Together with the loss-of-function studies, these data suggest that the levels of Sp1 determine the degree of p75NTR induction. Sp1 mRNA levels do not change in cells exposed to hypo-osmolarity, and through pulse-chase analysis, we demonstrated that the rate of Sp1 degradation is strongly inhibited in cells exposed to hypo-osmolarity. Together, these data suggest that hypo-osmolarity reduces Sp1 degradation and thereby elevates cellular Sp1 levels and promotes transcription from the p75NTR promoter. Interestingly, Peterson and Bogenmann (2003) reported that inhibitors of PKC and PLC (both used on GOTO cells) and NOS (used on A875 cells) reduce the accumulation of luciferase driven from a p75NTR promoter construct after exposure of cells to hypo-osmolarity. The different effects of PLC and NOS inhibitors in their systems and ours likely reflect cell type-specific responses to hypo-osmolarity and the differences in the outputs examined (p75NTR and Sp1 from endogenous genes vs luciferase activity from a p75NTR promoter construct).

How does hypo-osmolarity reduce the degradation of Sp1 levels? Previous studies have shown that $\mathrm{Sp} 1$ is subject to several post-translational modifications, including phosphorylation, acetylation, and glycosylation, and that Sp1 is degraded though the proteosome (Su et al., 1999; Bouwman and Philipsen, 2002; Li et al., 2004). We have shown that the effect of hypo-osmolarity on Sp1 levels requires both the PI3K and PKC pathways, and our working hypothesis is that these pathways alter post-translational modifications of Sp1 and thereby modulate its stability. Identifying the precise means by which $S p 1$ stability is regulated will allow us to determine whether these mechanisms function in vivo to alter Sp1 activity and p75NTR levels after injury to the intact nervous system.

\section{References}

Andsberg G, Kokaia Z, Lindvall O (2001) Upregulation of p75 neurotrophin receptor after stroke in mice does not contribute to differential vulnerability of striatal neurons. Exp Neurol 169:351-363.

Bhakar AL, Howell JL, Paul CE, Salehi AH, Becker EB, Said F, Bonni A, Barker PA (2003) Apoptosis induced by p75NTR overexpression requires Jun kinase-dependent phosphorylation of Bad. J Neurosci 23:11373-11381.

Birnbaum MJ, van Wijnen AJ, Odgren PR, Last TJ, Suske G, Stein GS, Stein JL (1995) Sp1 trans-activation of cell cycle regulated promoters is selectively repressed by Sp3. Biochemistry 34:16503-16508

Bouwman P, Philipsen S (2002) Regulation of the activity of Sp1-related transcription factors. Mol Cell Endocrinol 195:27-38.

Caprini M, Gomis A, Cabedo H, Planells-Cases R, Belmonte C, Viana F, Ferrer-Montiel A (2003) GAP43 stimulates inositol trisphosphatemediated calcium release in response to hypotonicity. EMBO J 22:3004-3014.
Casha S, Yu WR, Fehlings MG (2001) Oligodendroglial apoptosis occurs along degenerating axons and is associated with FAS and p75 expression following spinal cord injury in the rat. Neuroscience 103:203-218.

Ceni C, Barker PA (2005) Getting RIP'd stunts your growth. Neuron 46:839-840.

Copray JC, Jaarsma D, Kust BM, Bruggeman RW, Mantingh I, Brouwer N, Boddeke HW (2003) Expression of the low affinity neurotrophin receptor p75 in spinal motoneurons in a transgenic mouse model for amyotrophic lateral sclerosis. Neuroscience 116:685-694.

Costantini C, Scrable H, Puglielli L (2006) An aging pathway controls the TrkA to p75(NTR) receptor switch and amyloid beta-peptide generation. EMBO J 25:1997-2006.

Dickmeis T, Muller F (2005) The identification and functional characterization of conserved regulatory elements in developmental genes. Brief Funct Genomic Proteomic 3:332-350.

Farhadi HF, Lepage P, Forghani R, Friedman HC, Orfali W, Jasmin L, Miller W, Hudson TJ, Peterson AC (2003) A combinatorial network of evolutionarily conserved myelin basic protein regulatory sequences confers distinct glial-specific phenotypes. J Neurosci 23:10214-10223.

Fischer R, Schliess F, Haussinger D (1997) Characterization of the hypoosmolarity-induced $\mathrm{Ca} 2+$ response in cultured rat astrocytes. Glia 20:51-58.

Hershko DD, Robb BW, Wray CJ, Luo GJ, Hasselgren PO (2004) Superinduction of IL- 6 by cycloheximide is associated with mRNA stabilization and sustained activation of p38 map kinase and NF-kappaB in cultured caco-2 cells. J Cell Biochem 91:951-961.

Huber LJ, Chao MV (1995) Mesenchymal and neuronal cell expression of the p75 neurotrophin receptor gene occur by different mechanisms. Dev Biol 167:227-238.

Husson A, Quillard M, Fairand A, Chedeville A, Lavoinne A (1996) Hypoosmolarity and glutamine increased the beta-actin gene transcription in isolated rat hepatocytes. FEBS Lett 394:353-355.

King VR, Bradbury EJ, McMahon SB, Priestley JV (2000) Changes in truncated trkB and $\mathrm{p} 75$ receptor expression in the rat spinal cord following spinal cord hemisection and spinal cord hemisection plus neurotrophin treatment. Exp Neurol 165:327-341.

Kokaia Z, Andsberg G, Martinez-Serrano A, Lindvall O (1998) Focal cerebral ischemia in rats induces expression of P75 neurotrophin receptor in resistant striatal cholinergic neurons. Neuroscience 84:1113-1125.

Li L, He S, Sun JM, Davie JR (2004) Gene regulation by Sp1 and Sp3. Biochem Cell Biol 82:460-471.

Li T, Chen YH, Liu TJ, Jia J, Hampson S, Shan YX, Kibler D, Wang PH (2003) Using DNA microarray to identify $\mathrm{Sp} 1$ as a transcriptional regulatory element of insulin-like growth factor 1 in cardiac muscle cells. Circ Res 93:1202-1209.

Lopez SM, Perez-Perez M, Marquez JM, Naves FJ, Represa J, Vega JA (1998) p75 and TrkA neurotrophin receptors in human skin after spinal cord and peripheral nerve injury, with special reference to sensory corpuscles. Anat Rec 251:371-383.

Majdan M, Lachance C, Gloster A, Aloyz R, Zeindler C, Bamji S, Bhakar A, Belliveau D, Fawcett J, Miller FD, Barker PA (1997) Transgenic mice expressing the intracellular domain of the p75 neurotrophin receptor undergo neuronal apoptosis. J Neurosci 17:6988-6998.

Majello B, De Luca P, Lania L (1997) Sp3 is a bifunctional transcription regulator with modular independent activation and repression domains. J Biol Chem 272:4021-4026.

Meisse D, Renouf S, Husson A, Lavoinne A (1998) Cell swelling increased the alpha2-macroglobulin gene expression in cultured rat hepatocytes. FEBS Lett 422:346-348.

Michalke M, Cariers A, Schliess F, Haussinger D (2000) Hypoosmolarity influences the activity of transcription factor NF-kappaB in rat H4IIE hepatoma cells. FEBS Lett 465:64-68.

Nobrega MA, Pennacchio LA (2004) Comparative genomic analysis as a tool for biological discovery. J Physiol (Lond) 554:31-39.

Pang RT, Lee LT, Ng SS, Yung WH, Chow BK (2004) CpG methylation and transcription factors Sp1 and Sp3 regulate the expression of the human secretin receptor gene. Mol Endocrinol 18:471-483.

Papadopoulos MC, Saadoun S, Binder DK, Manley GT, Krishna S, Verkman AS (2004) Molecular mechanisms of brain tumor edema. Neuroscience 129:1011-1020.

Patil N, Lacy E, Chao MV (1990) Specific neuronal expression of human 
NGF receptors in the basal forebrain and cerebellum of transgenic mice. Neuron 4:437-447.

Paul CE, Vereker E, Dickson KM, Barker PA (2004) A pro-apoptotic fragment of the p75 neurotrophin receptor is expressed in p75NTRExonIV null mice. J Neurosci 24:1917-1923.

Peterson S, Bogenmann E (2003) Osmotic swelling induces p75 neurotrophin receptor (p75NTR) expression via nitric oxide. J Biol Chem 278:33943-33950.

Roux PP, Barker PA (2002) Neurotrophin signaling through the p75 neurotrophin receptor. Prog Neurobiol 67:203-233.

Roux PP, Colicos MA, Barker PA, Kennedy TE (1999) p75 neurotrophin receptor expression is induced in apoptotic neurons after seizure. J Neurosci 19:6887-6896.

Sehgal A, Patil N, Chao M (1988) A constitutive promoter directs expression of the nerve growth factor receptor gene. Mol Cell Biol $8: 3160-3167$.
Shaw G, Morse S, Ararat M, Graham FL (2002) Preferential transformation of human neuronal cells by human adenoviruses and the origin of HEK 293 cells. FASEB J 16:869-871.

Su K, Roos MD, Yang X, Han I, Paterson AJ, Kudlow JE (1999) An $\mathrm{N}$-terminal region of Sp1 targets its proteasome-dependent degradation in vitro. J Biol Chem 274:15194-15202.

Troy CM, Friedman JE, Friedman WJ (2002) Mechanisms of p75-mediated death of hippocampal neurons. Role of caspases. J Biol Chem 277:34295-34302.

Warskulat U, Kreuels S, Muller HW, Haussinger D (2001) Identification of osmosensitive and ammonia-regulated genes in rat astrocytes by Northern blotting and differential display reverse transcriptase-polymerase chain reaction. J Hepatol 35:358-366.

Wong WK, Chen K, Shih JC (2003) Decreased methylation and transcription repressor Sp3 up-regulated human monoamine oxidase (MAO) B expression during Caco-2 differentiation. J Biol Chem 278:36227-36235. 